\title{
Field Inhomogeneity Correction Based on Gridding Reconstruction for Magnetic Resonance Imaging
}

\author{
Holger Eggers*, Tobias Knopp, and Daniel Potts
}

\begin{abstract}
Spatial variations of the main field give rise to artifacts in magnetic resonance images if disregarded in reconstruction. With non-Cartesian $k$-space sampling, they often lead to unacceptable blurring. Data from such acquisitions are usually reconstructed with gridding methods and optionally restored with various correction methods. Both types of methods essentially face the same basic problem of adequately approximating an exponential function to enable an efficient processing with fast Fourier transforms. Nevertheless, they have commonly addressed it differently so far. In the present work, a unified approach is pursued. The principle behind gridding methods is first generalized to nonequispaced sampling in both domains and then applied to field inhomogeneity correction. Three new algorithms, which are compatible with a direct conjugate phase and an iterative algebraic reconstruction, are derived in this way from a straightforward embedding of the data into a higher dimensional space. Their evaluation in simulations and phantom experiments with spiral $k$-space sampling shows that one of them promises to provide a favorable compromise between fidelity and complexity compared with existing algorithms. Moreover, it allows a simple choice of key parameters involved in approximating an exponential function and a balance between the accuracy of reconstruction and correction.
\end{abstract}

Index Terms-Conjugate phase reconstruction, field inhomogeneity, gridding, image reconstruction, iterative reconstruction, magnetic resonance imaging, off-resonance correction, spiral imaging.

\section{INTRODUCTION}

M AGNETIC resonance imaging (MRI) relies on a strong, homogeneous main field. While the field strength determines the net magnetization available for signal generation, the field homogeneity ensures adequate coherence between the precession of individual spins within one voxel and thus sufficient signal lifetime for an efficient detection. More subtle variations of the field strength between different voxels lead to a distortion of the Fourier encoding used to spatially resolve the received signal and, without correction, to artifacts in images. These artifacts are mainly limited to geometric distortion and intensity variation for Cartesian acquisitions, which sample the spatial frequency domain of the images, the so-called $k$-space, on a

Manuscript received October 13, 2006; revised December 11, 2006. Asterisk indicates corresponding author.

*H. Eggers is with Philips Research Europe, Sector Medical Imaging Systems, 22335 Hamburg, Germany (e-mail: holger.eggers@ philips.com).

T. Knopp is with the Institute of Mathematics, University of Lübeck, 23560 Lübeck, Germany (e-mail: knopp@informatik.uni-luebeck.de).

D. Potts is with the Department of Mathematics, Chemnitz University of Technology, 09107 Chemnitz, Germany (e-mail: potts@mathematik.tu-chemnitz.de).

Digital Object Identifier 10.1109/TMI.2006.891502
Cartesian grid. For non-Cartesian acquisitions, however, more severe blurring and other artifacts arise.

Field inhomogeneity is in non-Cartesian imaging usually compensated by either a direct conjugate phase reconstruction (CPR) [1] or an iterative algebraic reconstruction [2]. Both are closely related, since one iteration of the latter typically involves one application of the former and its adjoint. The individual methods mainly differ in how they make the distortion of the Fourier encoding amenable to a processing with fast Fourier transforms (FFTs). They all introduce for this purpose a coarse segmentation, i.e., discretization, in either the time domain [1] or the frequency domain [3] and require for each resulting segment a separate transformation. In the respective other domain, they perform an interpolation to improve accuracy, such as a Hanning interpolation [1] or more sophisticated linear combinations [4], [5].

If field inhomogeneity is disregarded, non-Cartesian acquisitions are commonly reconstructed with gridding methods [6]. These first convolve the nonequispaced $k$-space samples with a window function of finite extent and resample them to an oversampled Cartesian grid to then employ FFTs for the transformation to image space. They also include a weighting of both the original samples and the resulting images to compensate for variations in sampling density and to counteract the effect of the convolution. Remarkably, they do not attempt to estimate the spatial frequency spectrum of the final images on the Cartesian grid. Gridding methods have been demonstrated to reach a better compromise between accuracy and complexity than simpler methods that rely on an interpolation in $k$-space only. Alternatively, nonuniform FFTs (NFFTs) have been considered for the transformation of the nonequispaced $k$-space samples to image space [7].

In fact, the same basic problem of adequately approximating an exponential function to enable an efficient processing with FFTs underlies the field inhomogeneity correction and the reconstruction of non-Cartesian acquisitions. Nevertheless, it has been addressed differently so far. Only recently, the similarity between both has been realized and exploited to suggest a conceptually very simple approach to field inhomogeneity correction [8]. By embedding the $k$-space samples and the image pixels into higher dimensional spaces, the processing can essentially be reduced to a Fourier transform. To cope with the irregular sampling in both domains, the use of special NFFTs was advocated.

We start in the present work from this idea and derive less complex and more accurate approaches to field inhomogeneity correction from it, which still adhere to a unified treatment of reconstruction and correction. In the next section, the problem 
of reconstructing images from non-Cartesian acquisitions in the presence of field inhomogeneity is described mathematically. The approximation that NFFTs are based on is then generalized to irregular sampling in both domains. With its help, three new algorithms for field inhomogeneity correction are derived from the proposed embedding of the data into higher dimensional spaces. They are evaluated in simulations and phantom experiments and compared against existing algorithms. Finally, their advantages and disadvantages are discussed.

\section{THEORY}

In MRI, the demodulated signal $s(t)$ received from an object with a magnetization $m(\boldsymbol{r})$ at a reference time point $t=0$ is ideally given by

$$
s(t)=\int_{\mathbb{R}^{3}} m(\boldsymbol{r}) \mathrm{e}^{-\mathrm{i} \boldsymbol{k}(t) \cdot \boldsymbol{r}} \mathrm{d} \boldsymbol{r} .
$$

$\boldsymbol{k}(t)$ denotes the trajectory, along which samples are acquired in $k$-space. It is determined by the time variant gradient field applied during the measurement.

Any inhomogeneity of the main field distorts the Fourier encoding that (1) describes. Taking this imperfection into account, $s(t)$ is more accurately modelled by

$$
s(t)=\int_{\mathbb{R}^{3}} m(\boldsymbol{r}) \mathrm{e}^{-\mathrm{i} \omega(\boldsymbol{r}) t} \mathrm{e}^{-\mathrm{i} \boldsymbol{k}(t) \cdot \boldsymbol{r}} \mathrm{d} \boldsymbol{r} .
$$

$\omega(\boldsymbol{r})$ denotes the angular off-resonance frequency, which is proportional to the local deviation of the field strength. Other imperfections, such as relaxation, are not considered in this work.

We restrict ourselves to 2-D imaging from now on. The sampled area of $k$-space is then confined to $k \in[-\pi, \pi]^{2}$, and the covered field of view to $\boldsymbol{r} \in\left[-\left(N_{1} / 2\right),\left(N_{1} / 2\right)\right] \times$ $\left[-\left(N_{2} / 2\right),\left(N_{2} / 2\right)\right]$. Discretizing the integral in (2) on $N_{1} N_{2}$ equispaced voxel positions $\boldsymbol{r}_{\rho}$ and the signal $s(t)$ on $M$ time points $t_{\kappa}$ yields

$$
s_{\kappa} \approx \tilde{s}_{\kappa}:=\sum_{\rho=0}^{N_{1} N_{2}-1} m_{\rho} \mathrm{e}^{-\mathrm{i} \omega_{\rho} t_{\kappa}} \mathrm{e}^{-\mathrm{i} \boldsymbol{k}_{\kappa} \cdot \boldsymbol{r}_{\rho}}
$$

where $s_{\kappa}:=s\left(t_{\kappa}\right), \tilde{s}_{\kappa}:=\tilde{s}\left(t_{\kappa}\right), m_{\rho}:=m\left(\boldsymbol{r}_{\rho}\right), \omega_{\rho}:=\omega\left(\boldsymbol{r}_{\rho}\right)$, and $k_{\kappa}:=k\left(t_{\kappa}\right)$. Using the vectors

$$
\begin{aligned}
\boldsymbol{s} & :=\left(s_{\kappa}\right)_{\kappa=0, \ldots, M-1} \\
\boldsymbol{m} & :=\left(m_{\rho}\right)_{\rho=0, \ldots, N_{1} N_{2}-1}
\end{aligned}
$$

and the matrix

$$
\boldsymbol{H}:=\left(\mathrm{e}^{-\mathrm{i} \omega_{\rho} t_{\kappa}} \mathrm{e}^{-\mathrm{i} \boldsymbol{k}_{\kappa} \cdot \boldsymbol{r}_{\rho}}\right)_{\kappa=0, \ldots, M-1 ; \rho=0, \ldots, N_{1} N_{2}-1}
$$

this may be rewritten as
We propose determining $\boldsymbol{m}$ by a weighted least squares approach

$$
\|\boldsymbol{s}-\boldsymbol{H} \boldsymbol{m}\|_{\boldsymbol{W}}=\sqrt{\sum_{\kappa=0}^{M-1} w_{\kappa}\left|s_{\kappa}-\tilde{s}_{\kappa}\right|^{2}} \stackrel{m}{\rightarrow} \min
$$

with factors $w_{\kappa}>0$ that compensate for variations in the local sampling density. It leads to the weighted normal equation of first kind

$$
\boldsymbol{H}^{\mathrm{H}} \boldsymbol{W} \boldsymbol{H} \boldsymbol{m}=\boldsymbol{H}^{\mathrm{H}} \boldsymbol{W} \boldsymbol{s}
$$

where $\boldsymbol{W}$ is a diagonal matrix with entries $\boldsymbol{W}_{\kappa \kappa}=w_{\kappa}$. Due to the size of this linear system, we suggest solving it iteratively with a suitable variant of the conjugate gradient method, such as the conjugate gradient normal equation residual (CGNR) method. In this way, (4) is in each iteration minimized over a certain Krylov space. Moreover, by choosing a zero vector as initial estimate of $\boldsymbol{m}$, the intermediate result after one iteration is, except for a scaling factor, identical to the right-hand side of this linear system and thus to the result of the CPR, which is one reason for including $\boldsymbol{W}$ in (5).

The computational complexity of determining $\boldsymbol{m}$ then depends primarily on two factors, the required number of iterations and the required effort per iteration. The first factor is mostly linked to the employed initial estimate of $\boldsymbol{m}$ and to the condition of $\boldsymbol{H}$. We stick with a zero vector as initial estimate and apply no additional preconditioning in this work, yet the presence of $\boldsymbol{W}$ in (5) is also motivated by its beneficial effect on the condition of the system matrix. The second factor is mainly influenced by the multiplication of a vector with the matrix $\boldsymbol{H}$ or $\boldsymbol{H}^{\mathrm{H}}$. Due to the distortion of the Fourier encoding, these products may not simply be implemented with FFTs. They may, however, efficiently be realized with NFFTs, as we show in the remainder of this section.

Following [8], we start by embedding the data in both domains in a higher dimensional space. For this purpose, we set $\boldsymbol{k}_{\kappa}^{\prime}:=\left(\left(\boldsymbol{k}_{\kappa}\right)^{\top}, t_{\kappa}\right)^{\top}$ and $\boldsymbol{r}_{\rho}^{\prime}:=\left(\left(\boldsymbol{r}_{\rho}\right)^{\top}, \omega_{\rho}\right)^{\top}$, i.e., we add a time dimension to the spatial frequency domain and an off-resonance frequency dimension to the spatial domain. Equation (3) may then be rewritten as

$$
s_{\kappa} \approx \sum_{\rho=0}^{N_{1} N_{2}-1} m_{\rho} \mathrm{e}^{-\mathrm{i} \boldsymbol{k}_{\kappa}^{\prime} \cdot \boldsymbol{r}_{\rho}^{\prime}} .
$$

The samples in both domains are now nonequispaced.

The NFFT and its adjoint, which are also known as nonuniform FFT of type 1 and type 2, are summarized in Appendix A. They require the samples in one domain to be equispaced. Unlike these standard NFFTs, a so-called NNFFT, or nonuniform FFT of type 3, was first suggested in [9] and later treated in more detail in [10], which permits the fast calculation of the Fourier transform of a vector of nonequispaced samples at a vector of nonequispaced positions. It basically constitutes a combination of the NFFT and its adjoint. Applying it to the computation of (6), an approach we will call 3-D NNFFT, entails a 3-D FFT

$$
s \approx H \boldsymbol{m} .
$$


and in both domains a 3-D local linear combination. Additionally, it demands two multiplicative oversampling factors, which increase the length of the Fourier transform for all three dimensions. The evaluation of the adjoint of (6), i.e., of the sum

$$
\sum_{\kappa=0}^{M-1} s_{\kappa} \mathrm{e}^{\mathrm{i} \boldsymbol{k}_{\kappa}^{\prime} \cdot \boldsymbol{r}_{\rho}^{\prime}}
$$

involves the same effort.

To use the standard NFFTs instead, we have to resample the data in one domain to a Cartesian grid. Preferably, the spatial domain is chosen, since it involves no effort for $\boldsymbol{r}$. We employ the approximation

$$
\mathrm{e}^{2 \pi \mathrm{i} k x} \approx \frac{1}{\alpha N \hat{\varphi}(x)} \sum_{l=-\alpha N / 2}^{\alpha N / 2-1} \psi\left(k-\frac{l}{\alpha N}\right) \mathrm{e}^{2 \pi \mathrm{i} \frac{l x}{\alpha N}}
$$

for this purpose, with a real oversampling factor $\alpha$, an integer constant $N$, and the Fourier transform $\hat{\varphi}(x)$ and the truncation $\psi(k)$ of a window function $\varphi(k)$ with a kernel size of $2 \mu$, where $k \in[-(1 / 2)+(\mu / \alpha N),(1 / 2)-(\mu / \alpha N)]$ and $x \in[-(N / 2),(N / 2)]$. It is closely related to the approximation underlying the standard NFFTs (A-4) and derived in Appendix B. Moreover, we choose an integer constant $N_{3}$ such that

$$
\frac{\omega_{\rho} t_{\kappa}}{2 \pi} \in\left[-\frac{N_{3}}{4}+\frac{\mu}{2 \alpha}, \frac{N_{3}}{4}-\frac{\mu}{2 \alpha}\right]
$$

for all $\rho$ and $\kappa$, and a scaling factor $W$ such that

$$
\frac{\omega_{\rho}}{W} \in\left[-\frac{1}{2}+\frac{\mu}{\alpha N_{3}}, \frac{1}{2}-\frac{\mu}{\alpha N_{3}}\right]
$$

for all $\rho$. As the computational complexity grows with it, $N_{3}$ should be kept as small as possible. Centering $\omega_{\rho}$ and $t_{\kappa}$, which involves in both domains a multiplication with a phase, is, therefore, advantageous. We then obtain with (8)

$$
\begin{aligned}
\mathrm{e}^{-\mathrm{i} \omega_{\rho} t_{\kappa}}= & \mathrm{e}^{-2 \pi \mathrm{i} \frac{\omega_{\rho}}{W} \frac{W t_{\kappa}}{2 \pi}} \\
\approx & \frac{1}{\alpha N_{3} \hat{\varphi}\left(-\frac{W t_{\kappa}}{2 \pi}\right)} \\
& \times \sum_{l=-\alpha N_{3} / 2}^{\alpha N_{3} / 2-1} \psi\left(\frac{\omega_{\rho}}{W}-\frac{l}{\alpha N_{3}}\right) \mathrm{e}^{-\mathrm{i} \frac{W t_{\kappa} l}{\alpha N_{3}}}
\end{aligned}
$$

and by insertion in (3)

$$
\begin{array}{r}
s_{\kappa} \approx \frac{1}{\alpha N_{3} \hat{\varphi}\left(-\frac{W t_{\kappa}}{2 \pi}\right)} \sum_{l=-\alpha N_{3} / 2}^{\alpha N_{3} / 2-1} \sum_{\rho=0}^{N_{1} N_{2}-1} m_{\rho} \psi\left(\frac{\omega_{\rho}}{W}-\frac{l}{\alpha N_{3}}\right) \\
\times \mathrm{e}^{-\mathrm{i} \boldsymbol{k}_{\kappa} \cdot \boldsymbol{r}_{\rho}} \mathrm{e}^{-\mathrm{i} \frac{W t_{\kappa} l}{\alpha N_{3}}} .
\end{array}
$$

Setting $\boldsymbol{k}_{\kappa}^{\prime \prime}:=\left(\left(\boldsymbol{k}_{\kappa}\right)^{\top}, W t_{\kappa} /\left(\alpha N_{3}\right)\right)^{\top}$ and $\boldsymbol{r}_{(\rho, l)}^{\prime \prime}:=$ $\left(\left(\boldsymbol{r}_{\rho}\right)^{\top}, l\right)^{\top}$ finally yields

$$
\begin{aligned}
s_{\kappa} \approx \frac{1}{\alpha N_{3} \hat{\varphi}\left(-\frac{W t_{\kappa}}{2 \pi}\right)} \sum_{l=}^{\alpha N_{3} / 2-1} \sum_{\rho=0}^{N_{1} N_{2}-1} m_{\rho} \\
\quad \times \psi\left(\frac{\omega_{\rho}}{W}-\frac{l}{\alpha N_{3}}\right) \mathrm{e}^{-\mathrm{i} \boldsymbol{k}_{\kappa}^{\prime \prime} \cdot \boldsymbol{r}_{(\rho, l)}^{\prime \prime}} .
\end{aligned}
$$

The matrix-vector product may thus be realized by a 3-D NFFT. Hence, we will call this approach 3-D NFFT. It requires a 3-D FFT and a 3-D local linear combination in the spatial frequency domain, and it introduces two multiplicative oversampling factors only for the added third dimension. The adjoint reads

$$
\sum_{l=-\alpha N_{3} / 2}^{\alpha N_{3} / 2-1} \psi\left(\frac{\omega_{\rho}}{W}-\frac{l}{\alpha N_{3}}\right) \sum_{\kappa=0}^{M-1} \frac{s_{\kappa}}{\alpha N_{3} \hat{\varphi}\left(\frac{W t_{\kappa}}{2 \pi}\right)} \mathrm{e}^{\mathrm{i} \boldsymbol{k}_{\kappa}^{\prime \prime} \cdot \boldsymbol{r}_{(\rho, l)}^{\prime \prime}}
$$

and may also be computed with a 3-D NFFT, but additionally with a sparse summation over $l$.

We now consider separating the 3-D domains into 2-D $\otimes 1-\mathrm{D}$ domains. In this way, the Fourier transform along the added third dimension can be replaced by an explicit sum, which appears beneficial in view of the sparseness of the data in the 3-D spaces. By merely rearranging (10), we obtain

$$
\begin{aligned}
s_{\kappa} \approx & \sum_{l=-\alpha N_{3} / 2}^{\alpha N_{3} / 2-1} \frac{\mathrm{e}^{-\mathrm{i} \frac{W t_{\kappa} l}{\alpha N_{3}}}}{\alpha N_{3} \hat{\varphi}\left(-\frac{W t_{\kappa}}{2 \pi}\right)} \\
& \times \sum_{\rho=0}^{N_{1} N_{2}-1} m_{\rho} \psi\left(\frac{\omega_{\rho}}{W}-\frac{l}{\alpha N_{3}}\right) \mathrm{e}^{-\mathrm{i} \boldsymbol{k}_{\kappa} \cdot \boldsymbol{r}_{\rho}} .
\end{aligned}
$$

With this approximation, the matrix-vector product may be calculated by $\alpha N_{3}$ 2-D NFFTs and a summation over $l$, an approach we will call 2-D $\otimes 1$-D NFFT-F. It involves a 2-D FFT and a 2-D local linear combination in the spatial frequency domain for each NFFT, and it demands only one oversampling factor for each dimension. The adjoint is given by

$$
\sum_{l=-\alpha N_{3} / 2}^{\alpha N_{3} / 2-1} \psi\left(\frac{\omega_{\rho}}{W}-\frac{l}{\alpha N_{3}}\right) \sum_{\kappa=0}^{M-1} \frac{s_{\kappa} \mathrm{e}^{\mathrm{i} \frac{W t_{\kappa} l}{\alpha N_{3}}}}{\alpha N_{3} \hat{\varphi}\left(\frac{W t_{\kappa}}{2 \pi}\right)} \mathrm{e}^{\mathrm{i} \boldsymbol{k}_{\kappa} \cdot \boldsymbol{r}_{\rho}}
$$

where the summation over $l$ is sparse.

We derive a variant of this approach by defining another scaling factor $T$ such that

$$
\frac{t_{\kappa}}{T} \in\left[-\frac{1}{2}+\frac{\mu}{\alpha N_{3}}, \frac{1}{2}-\frac{\mu}{\alpha N_{3}}\right]
$$

for all $\kappa$. Using again the approximation (B-1), we get

$$
\begin{aligned}
\mathrm{e}^{-\mathrm{i} \omega_{\rho} t_{\kappa}}= & \mathrm{e}^{-2 \pi \mathrm{i} \frac{t_{\kappa}}{T} \frac{\omega_{\rho} T}{2 \pi}} \\
\approx & \frac{1}{\alpha N_{3} \hat{\varphi}\left(-\frac{\omega_{\rho} T}{2 \pi}\right)} \\
& \times \sum_{l=-\alpha N_{3} / 2}^{\alpha N_{3} / 2-1} \psi\left(\frac{t_{\kappa}}{T}-\frac{l}{\alpha N_{3}}\right) \mathrm{e}^{-\mathrm{i} \frac{\omega_{\rho} T l}{\alpha N_{3}}}
\end{aligned}
$$


and insertion into (3) yields

$$
\begin{aligned}
s_{\kappa} \approx & \sum_{l=-\alpha N_{3} / 2}^{\alpha N_{3} / 2-1} \psi\left(\frac{t_{\kappa}}{T}-\frac{l}{\alpha N_{3}}\right) \\
& \times \sum_{\rho=0}^{N_{1} N_{2}-1} \frac{m_{\rho} \mathrm{e}^{-\mathrm{i} \frac{\omega_{\rho} T l}{\alpha N_{3}}}}{\alpha N_{3} \hat{\varphi}\left(-\frac{\omega_{\rho} T}{2 \pi}\right)} \mathrm{e}^{-\mathrm{i} \boldsymbol{k}_{\kappa} \cdot \boldsymbol{r}_{\rho}} .
\end{aligned}
$$

In this way, the matrix-vector product may also be computed by $\alpha N_{3}$ 2-D NFFTs, followed by a sparse summation over $l$, an approach we will call 2-D $\otimes 1-\mathrm{D}$ NFFT-T. The effort for the NFFTs is substantially reduced if they are evaluated only at those $k$-space positions that actually contribute to the sparse summation. For the adjoint

$$
\sum_{l=-\alpha N_{3} / 2}^{\alpha N_{3} / 2-1} \frac{\mathrm{e}^{\mathrm{i} \frac{\omega_{\rho} T l}{\alpha N_{3}}}}{\alpha N_{3} \hat{\varphi}\left(\frac{\omega_{\rho} T}{2 \pi}\right)} \sum_{\kappa=0}^{M-1} s_{\kappa} \psi\left(\frac{t_{\kappa}}{T}-\frac{l}{\alpha N_{3}}\right) \mathrm{e}^{\mathrm{i} \boldsymbol{k}_{\kappa} \cdot \boldsymbol{r}_{\rho}}
$$

the summation over $l$ is no longer sparse, but the effort for the NFFTs may be decreased similarly.

With respect to computational complexity, we conclude that the 2-D $\otimes 1-D$ NFFT-T approach is the most efficient. It combines the advantages of an explicit summation and of an implicit local linear combination along the added third dimension in the spatial frequency domain. It is followed by the $2-\mathrm{D} \otimes$ 1-D NFFT-F and the 3-D NFFT approaches, which each share one of the advantages with it. The 3-D NNFFT approach is the least efficient, since it increases not only the number of segments from $\alpha N_{3}$ to $\alpha^{2} N_{3}$, like the 3-D NFFT approach, but also the length of the Fourier transform for the other two dimensions from $\alpha N_{1} \times \alpha N_{2}$ to $\alpha^{2} N_{1}+2 \alpha \mu \times \alpha^{2} N_{2}+2 \alpha \mu$.

\section{METHODS}

We integrated the four outlined gridding-based approaches into the CGNR method to solve (5). We then assessed them in simulations and phantom experiments using spiral $k$-space sampling. By stopping the CGNR method after either one or several iterations, we covered the cases of a direct CPR and an iterative algebraic reconstruction.

\section{A. Simulations}

As reference image, a Shepp-Logan phantom with a resolution of $256 \times 256$ was employed. We applied a slightly smoothed circular shutter with a radius of $7 / 8 \pi$ to it in $k$-space, since spiral acquisitions only sample a circular area in $k$-space and field inhomogeneity seemingly spreads the spatial frequency spectrum over time. The main field was modelled with the two field maps displayed in Fig. 1. The first reflects a continuous, parabolic variation of the field strength, the second a discrete, linear one. The rather unnatural discrete field map was chosen to study how the algorithms cope with a strong local variation of the field strength. Both maps span the same range of off-resonance frequencies of $-125 \mathrm{~Hz}$ to $+125 \mathrm{~Hz}$.

$k$-space data were calculated by a direct evaluation of (3). We segmented the acquisition into 12 spiral interleaves with 13332 samples each, including a twofold oversampling. Two images obtained with a standard gridding reconstruction of these data, i.e., without field inhomogeneity correction, are also presented in Fig. 1. An analytical function described in [11] served the sampling density compensation in this and all other cases presented in this work. Obviously, both field distributions give rise to blurring, but a strong local variation of the field strength causes further major artifacts.

The implementation of the 3-D NNFFT and 3-D NFFT approaches basically involves the interfacing to 3-D NNFFT and 3-D NFFT algorithms only. The 3-D NFFT approach additionally requires a postweighting of the data. The implementation of all 2-D approaches was based on the general framework outlined in [12]. It decomposes the approximation of (3) into three steps: 1) a preweighting of the data, 2) a transformation of each segment, and 3) a linear combination. Mathematically, it may be described by

$$
\begin{aligned}
m_{\rho, l}^{\prime} & :=v_{\rho, l} m_{\rho}, \\
s_{\kappa, l}^{\prime} & :=\sum_{\rho=0}^{N_{1} N_{2}-1} m_{\rho, l}^{\prime} \mathrm{e}^{-\mathrm{i} \boldsymbol{k}_{\kappa} \cdot \boldsymbol{r}_{\rho}} \\
s_{\kappa} & \approx \sum_{l=-\alpha N_{3} / 2}^{\alpha N_{3} / 2-1} w_{\kappa, l} s_{\kappa, l}^{\prime} .
\end{aligned}
$$

For the 2-D $\otimes 1-\mathrm{D}$ NFFT-T approach, for instance, the weights $v_{\rho, l}$ and $w_{\kappa, l}$ are given by

$$
\begin{aligned}
v_{\rho, l} & =\frac{\mathrm{e}^{-\mathrm{i} \frac{\omega_{\rho}^{\prime} T l}{\alpha N_{3}}-\mathrm{i} \omega_{\rho} t_{c}}}{\alpha N_{3} \hat{\varphi}\left(-\frac{\omega_{\rho}^{\prime} T}{2 \pi}\right)} \\
w_{\kappa, l} & =\psi\left(\frac{t_{\kappa}^{\prime}}{T}-\frac{l}{\alpha N_{3}}\right) \mathrm{e}^{-\mathrm{i} \omega_{c} t_{\kappa}^{\prime}}
\end{aligned}
$$

where $\omega_{c}$ and $t_{c}$ denote the central and $\omega^{\prime}$ and $t^{\prime}$ the centered angular off-resonance frequencies and time points, respectively. In the second step, only those $s_{\kappa, l}^{\prime}$ need to be computed with the $\alpha N_{3}$ NFFTs for which $w_{\kappa, l} \neq 0$. For all approaches, pruning techniques were applied to the FFTs that underlie the NFFTs to improve efficiency in view of the employed oversampling.

Besides these gridding-based approaches, a number of existing 2-D algorithms were implemented for comparison. These included a nearest neighbor interpolation with frequency segmentation [3], a Hanning interpolation with time segmentation [1], the more sophisticated Man interpolation with frequency segmentation [4], and a least squares interpolation with time segmentation [5]. Additionally, the counterparts with the respective other segmentation were implemented, exploiting the duality between time and frequency segmentation [12]. For the Man interpolation with time segmentation, a manual variation of the oversampling factor was performed, since no explicit rule for its choice was known. For the least squares interpolation, a Householder transformation was employed to solve the minimization problems.

All algorithms were programmed both in Matlab and in C. They were tested on a conventional workstation equipped with an Intel Xeon processor running at $2 \mathrm{GHz}$ and with $256 \mathrm{MB}$ of memory. The software configuration used was Linux 2.4.21, 

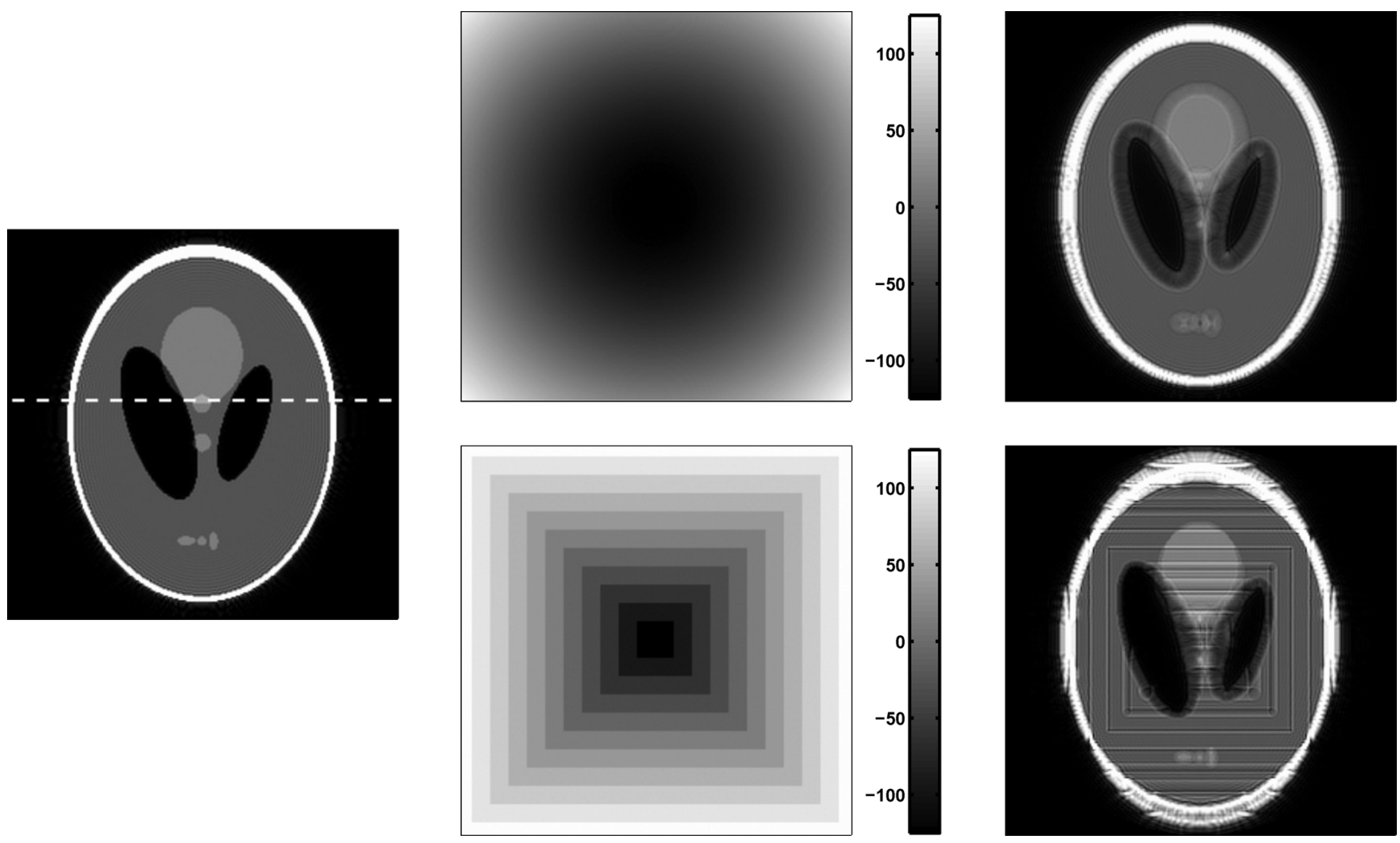

Fig. 1. Image and field maps used in simulations. Shown are a filtered Shepp-Logan phantom on the left, a continuous, parabolic and a discrete, linear field map with off-resonance frequencies in the range of $-125 \mathrm{~Hz}$ to $+125 \mathrm{~Hz}$ in the middle, and results of a standard gridding reconstruction on the right. The latter were obtained from simulations of spiral $k$-space sampling with a readout duration of $32 \mathrm{~ms}$. The dashed line superimposed on the phantom indicates the position where cross sections were taken for comparison.

FFTW 3.0.1, and NFFT 2.0. The latter is available from [13] and essentially differs from a standard gridding reconstruction in two respects only. Instead of a Kaiser-Bessel window, its Fourier transform is employed as window function, and its shape parameter is well defined. Images obtained with these algorithms were assessed both visually and quantitatively. For the latter, we used as measure the root of the sum of squares of the differences between reconstructed and original image pixels, divided by the root of the sum of squares of the original image pixels.

\section{B. Experiments}

The experiments were performed on a 1.5 T Achieva wholebody scanner (Philips Medical Systems, Best, The Netherlands). Transversal cross sections of standard imaging phantoms were acquired with a resolution of $256 \times 256$ pixels using a segmented spiral gradient echo sequence. A field-of-view of $250 \mathrm{~mm}$, a slice thickness of $10 \mathrm{~mm}$, a flip angle of $90^{\circ}$, a TE of $2 \mathrm{~ms}$, and a TR of $1 \mathrm{~s}$ were chosen. The readout duration, i.e., the length of time that data are acquired after each excitation, varied between $10 \mathrm{~ms}$ and $60 \mathrm{~ms}$, and the number of spiral interleaves changed accordingly.

Field maps were obtained from two separate measurements, which usually differed in TE by $1 \mathrm{~ms}$. Two images were reconstructed from them and thresholded based on signal intensity. Their phases were then subtracted and the differences scaled and slightly filtered. To reduce edge effects, the resulting field maps were additionally extrapolated to areas masked out before.

\section{RESULTS}

\section{A. Simulations}

The four gridding-based approaches are analyzed regarding their accuracy in Table I. The presented simulation results were obtained with the continuous field map and the settings $\alpha=$ $1.25, \mu=2$, and $\alpha N_{3}=14$. After one iteration, all four algorithms yield similar errors, i.e., they achieve a comparable accuracy for the case of a direct CPR. After three iterations, the 3 -D NNFFT approach produces an about $100 \%$ and the 2-D $\otimes$ 1-D NFFT-F approach an about $20 \%$ higher error than the two others. Beyond three iterations, errors did not decrease significantly anymore for any algorithm. These simulation results were found to be representative.

The running times of the four gridding-based approaches are compiled in Table II. As expected from the theoretical considerations, the 2-D $\otimes 1-D$ NFFT-T approach is the fastest and the 3-D NNFFT approach is the slowest. Remarkably, the 3-D NFFT approach clearly outperforms the 2-D $\otimes 1$-D NFFT-F approach, indicating that the locality of the linear combination along the added third dimension in the spatial frequency domain is dominant.

These two comparisons suggest that the 2-D $\otimes 1$-D NFFT-T approach is the preferred of the four gridding-based algorithms. We, therefore, selected it for all further investigations.

Results of its application to the images in Fig. 1 are presented in Fig. 2. For the continuous field map, already the first iteration 
TABLE I

COMPARISON OF THE ACCURACY OF DIFFERENT GRIDDING-BASED CORRECTION ALGORITHMS. LISTED IS THE NORMALIZED ROOT MEAN SQUARE (RMS) ERROR AFTER 1, 2, AND 3 ITERATIONS

\begin{tabular}{|c|c|c|c|}
\hline Iteration & 1 & 2 & 3 \\
\hline 3-D NNFFT & $5.41 \cdot 10^{-2}$ & $1.15 \cdot 10^{-2}$ & $1.13 \cdot 10^{-2}$ \\
\hline 3-D NFFT & $5.31 \cdot 10^{-2}$ & $5.83 \cdot 10^{-3}$ & $5.42 \cdot 10^{-3}$ \\
\hline $2-\mathrm{D} \otimes 1-\mathrm{D}$ NFFT-F & $5.39 \cdot 10^{-2}$ & $6.99 \cdot 10^{-3}$ & $6.39 \cdot 10^{-3}$ \\
\hline 2-D $\otimes 1-D$ NFFT-T & $5.32 \cdot 10^{-2}$ & $5.50 \cdot 10^{-3}$ & $5.21 \cdot 10^{-3}$ \\
\hline
\end{tabular}

TABLE II

COMPARISON OF THE RUNNING TIMES OF DIFFERENT GRIDDING-BASED CORRECTION Algorithms. Listed IS THE MEASUREd COMPUTATION TIME PER ITERATION

\begin{tabular}{|l||c|}
\hline \multicolumn{1}{|c||}{ Algorithm } & Running Time \\
\hline \hline 3-D NNFFT & $2000 \mathrm{~ms}$ \\
\hline 3-D NFFT & $1060 \mathrm{~ms}$ \\
\hline 2-D $\otimes 1$-D NFFT-F & $1530 \mathrm{~ms}$ \\
\hline 2-D $\otimes 1$-D NFFT-T & $840 \mathrm{~ms}$ \\
\hline
\end{tabular}

yields a visually good image. Mainly the second iteration provides further improvements, in particular at edges. For the discrete field map, the first iteration produces a visually unacceptable image due to artifacts arising from the strong local variation of the field strength. These artifacts are dramatically reduced by the second iteration, but only after about ten iterations the shape of the field map is no longer discernible in the image. This difference in the speed of convergence agrees with earlier work, which showed that the direct CPR performs reasonably well for spiral $k$-space sampling only if the field map is smooth [12], [14].

The accuracy of the 2-D $\otimes 1$-D NFFT-T approach is contrasted with that of existing algorithms in Fig. 3. The errors after one and three iterations were again obtained with the continuous field map and with the settings $\alpha=1.25$ and $\mu=2$ for the 2-D NFFTs. The results for the least squares algorithm were produced with a time segmentation. Using a frequency segmentation instead, errors increased for low number of segments and remained comparable for high number of segments. The results for the gridding-based approach involved matching the oversampling factor and the kernel size to the respective $\alpha N_{3}$. Errors are plotted down to the minimum number of segments defined by (9), which is 8 in this case. The results for the Man interpolation, for which the same lower bound holds, were obtained with a frequency interpolation. Slightly lower errors were achieved with a time segmentation, but the manual tuning of the oversampling factor rendered it impracticable. The results for the nearest neighbor interpolation are not shown, because it failed to yield acceptable images for more than one iteration at all. While the least squares interpolation obviously provides the lowest errors of all algorithms, the gridding-based approach reaches comparable levels very rapidly for sufficiently large oversampling factors and kernel sizes. Using the same settings as for the 2-D NFFTs, $\alpha N_{3}=14$ and both algorithms attain a similar accuracy. Moreover, errors do not decrease significantly anymore for higher number of segments, indicating that the 2-D NFFTs limit the overall accuracy from this point on. The Man interpolation provides reasonably good results after one iteration, but needs many segments to reach an adequate accuracy after three iterations. The Hanning interpolation performs poorly, except for very few segments.

The running times per iteration of the same algorithms are compiled in Table III for a fixed number of segments of 14 . Since they do not include the initialization, the differences are mainly due to varying amounts of data to be regridded per iteration. These amounts are determined by the number of non-zero weights in the linear combination. The least squares and the Man interpolation show the longest running times, since all $w_{\kappa, l} \neq 0$. By contrast, only a maximum of $2 \mu+1$ out of each $\alpha N_{3}$ weights are nonzero using the gridding-based approach, and only a maximum of 3 using the Hanning interpolation. Consequently, both exhibit significantly shorter running times.

\section{B. Experiments}

Representative results of the phantom experiments are summarized in Fig. 4. The off-resonance frequencies cover a range of $210 \mathrm{~Hz}$ in this example. Using 12 spiral interleaves and a readout duration of $28.5 \mathrm{~ms}$, the 2-D $\otimes 1$-D NFFT-T approach yields an almost perfect image after three iterations. The number of segments was 12 in this case, corresponding to $\alpha=1.33$. Using 6 spiral interleaves and a readout duration of $56.5 \mathrm{~ms}$, it still provides an image of good quality, although residual artifacts remain visible, mostly near the circumference and the resolution rods of the phantom. The number of segments was 19 in this case, corresponding to $\alpha=1.26$.

\section{DISCUSSION}

The efficient reconstruction of non-Cartesian acquisitions faces the problem of a nonequispaced sampling in the spatial frequency domain. It is adequately solved by both the adjoint NFFT and gridding reconstruction, which rely on the same approximation of an exponential function (A-4). Field inhomogeneity, however, introduces an exponential function with irregular sampling in both domains. We showed that (A-4) can be generalized to this case, leading to the similar approximation (B-1). In this way, the field inhomogeneity correction and the reconstruction of non-Cartesian acquisitions may be founded on the same basic approximation. Based on this concept, we derived three new algorithms in the present work.

Among the gridding-based algorithms, the 3-D NNFFT approach shows the highest error. We attribute this to the unnecessary interpolation for all spatial dimensions. As already pointed out in [6], gridding remains an approximation even if the samples are equispaced. The usual discretization of images on a Cartesian grid should, therefore, be exploited. The inferior accuracy of the 2-D $\otimes 1$-D NFFT-F approach compared to the 3-D 

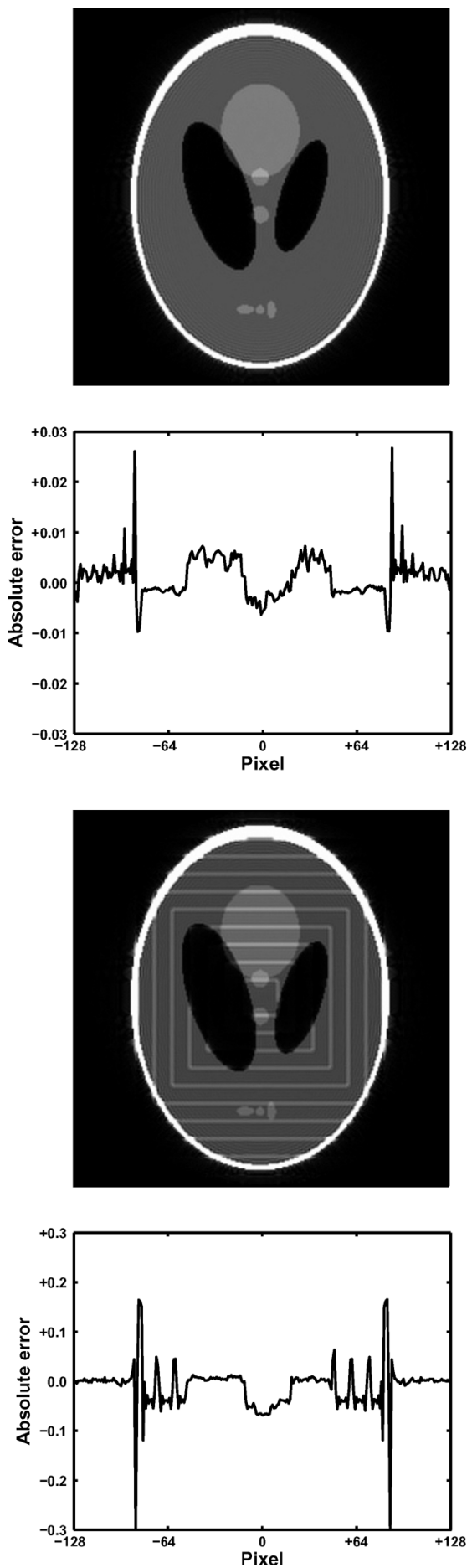
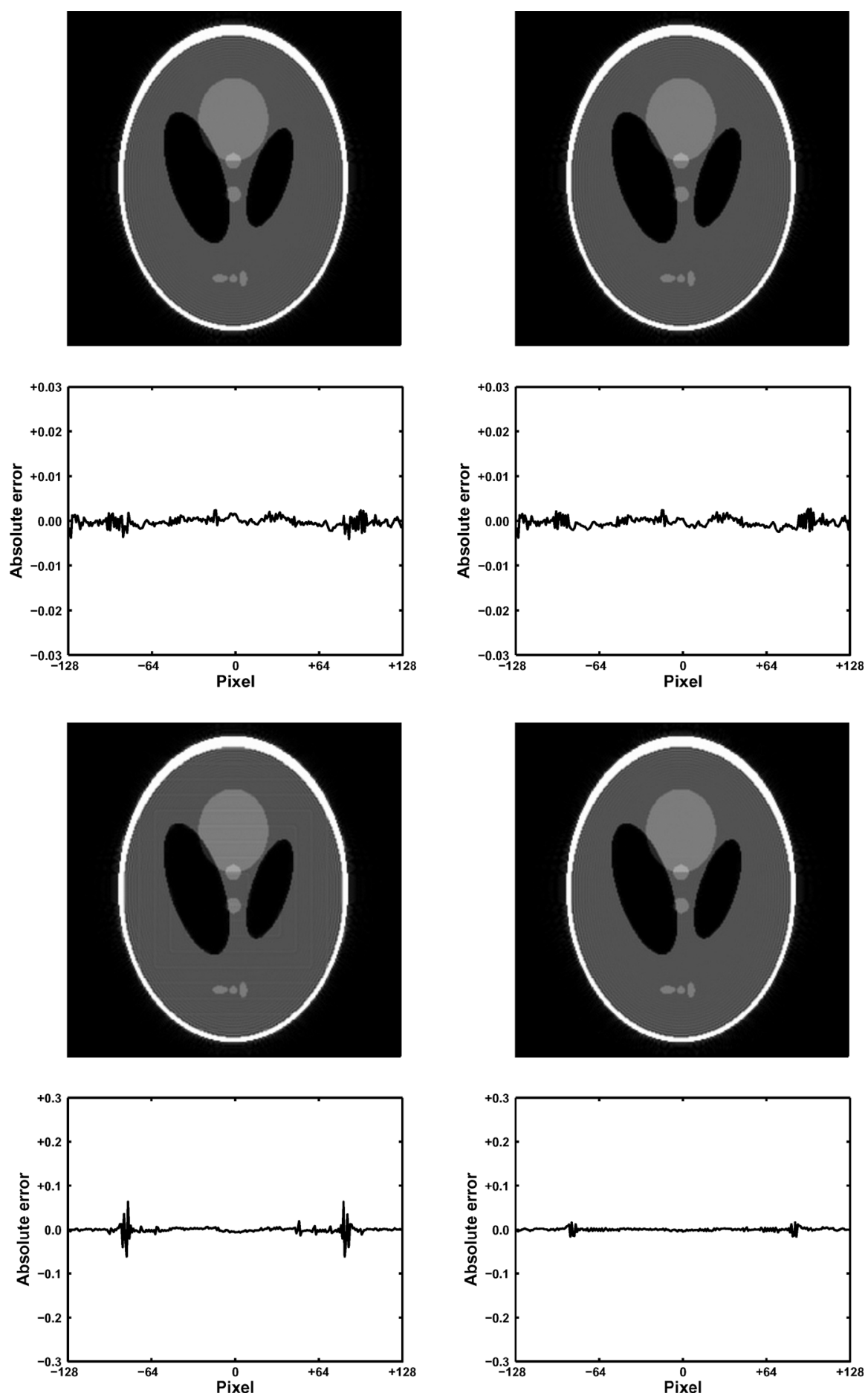

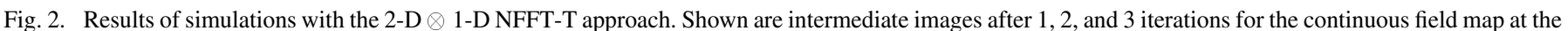

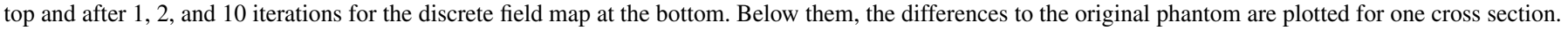
The scaling of the graphs varies by one order of magnitude between the top and the bottom.

NFFT and 2-D $\otimes 1-D$ NFFT-T approaches is in line with results obtained with the other, existing algorithms. Performing the interpolation in the transformed domain of the final result, i.e., in the spatial frequency domain of the images, generally decreased the artifact level, both visually and quantitatively. We selected the 2-D $\otimes 1-D$ NFFT-T approach for further investigations in this work for complexity reasons. However, it is worth noting that the 3-D NFFT approach achieves a similar accuracy while being conceptually simpler.
The results in Fig. 3 underline the advantage of using a local linear combination in conjunction with a weighting in the transformed domain instead of a local linear combination only, since the accuracy of the gridding-based approach is substantially superior to that of the Hanning interpolation from a certain minimum number of segments on. It then also exceeds the accuracy of the Man interpolation, which may be considered as a nonoptimal, global linear combination. Moreover, for a calculable, slightly higher number of segments, the 2-D NFFTs limit 

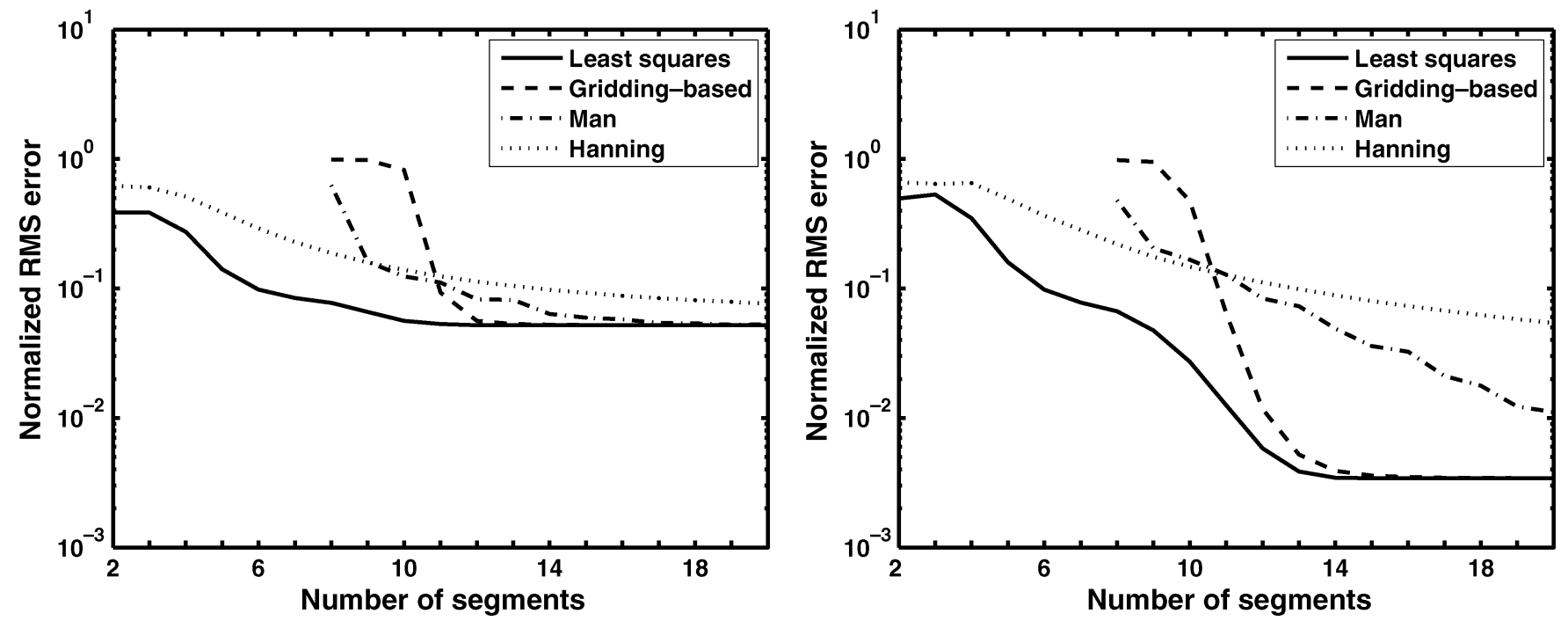

Fig. 3. Comparison of the accuracy of different correction algorithms. Plotted is the normalized root mean square (RMS ) error as function of the number of segments in the interpolation after 1 and 3 iterations on the left and on the right, respectively.

TABLE III

COMPARISON OF THE RUNNING TIMES OF DIFFERENT CORRECTION ALGORITHMS. LISTED IS THE MEASURED COMPUTATION TIME PER ITERATION, USING COMPARABLE PARAMETER SETTINGS

\begin{tabular}{|l||c|}
\hline \multicolumn{1}{|c||}{ Algorithm } & Running Time \\
\hline \hline Least squares & $1530 \mathrm{~ms}$ \\
\hline Gridding-based & $840 \mathrm{~ms}$ \\
\hline Man & $1530 \mathrm{~ms}$ \\
\hline Hanning & $710 \mathrm{~ms}$ \\
\hline
\end{tabular}

the achievable overall accurancy, thus equalizing it for the gridding-based approach and the least squares interpolation.

The results in Table III highlight the relevance of the amount of data to be regridded for the complexity. As in gridding reconstruction, the use of a local neighborhood in the interpolation appears to be advantageous. However, the primary benefit is in this case the reduced effort for the 2-D NFFTs rather than for the linear combination. Previously, it has been proposed to eliminate most of the regridding from both the direct CPR [12] and the iterative algebraic reconstruction [15]. While a detailed comparison remains to be done, the apparent advantage is often offset by either a restriction on the supported $k$-space trajectories or the requirement of higher oversampling factors to avoid excessive backfolding, as demonstrated for a related problem in parallel imaging [16].

The required number of segments also has a considerable influence on the complexity. For the gridding-based approach, the application of the well-known concept of oversampling permits a simple choice of an adequate number of segments. Moreover, the unified treatment of reconstruction and correction enables a balance of the accuracy of both. Thus, the accuracy of one is not increased beyond the limit set by the other. The same choice may, in principle, be used for the least squares interpolation. Fig. 3 suggests, however, that the error flattens out with fewer segments compared to the gridding-based approach. While this observation may be exploited to further reduce the complexity especially for the direct CPR in this particular case, it is not necessarily generalizable.

Finally, the effort involved in the initialization affects the complexity. It is mainly determined by the calculation of the weights for the linear combination from a given field map. Those approaches which require little such effort are clearly preferable for small number of iterations. The Hanning and the Man interpolation, but also the gridding-based approaches are among these, since they solely need to evaluate a given function. By contrast, the considerably higher effort for the least squares interpolation usually pays off for large number of iterations only.

The framework we chose for field inhomogeneity correction assumes a piecewise constant field map. While this is usually a sufficiently accurate model, a more precise one is obtained by also taking intravoxel gradients into account. These lead to a distortion of the actual $k$-space trajectory, which varies with the spatial position. For a more detailed description of the problem and a potential solution, the reader is referred to [17]. The framework we proposed for reconstruction includes a weighting in the linear system of equations to be solved. This weighting entails in theory a signal-to-noise (SNR) penalty [5]. In our experience, however, the loss in SNR was insignificant and was far outweighed by the acceleration of convergence it provided.

\section{APPENDIX A}

In this appendix, the NFFT and its adjoint, i.e., the nonuniform FFT of type 1 and type 2 [9], are outlined for the one dimensional case, and their close relation to gridding reconstruction is highlighted. For a more detailed description, the reader is referred to [18]. 

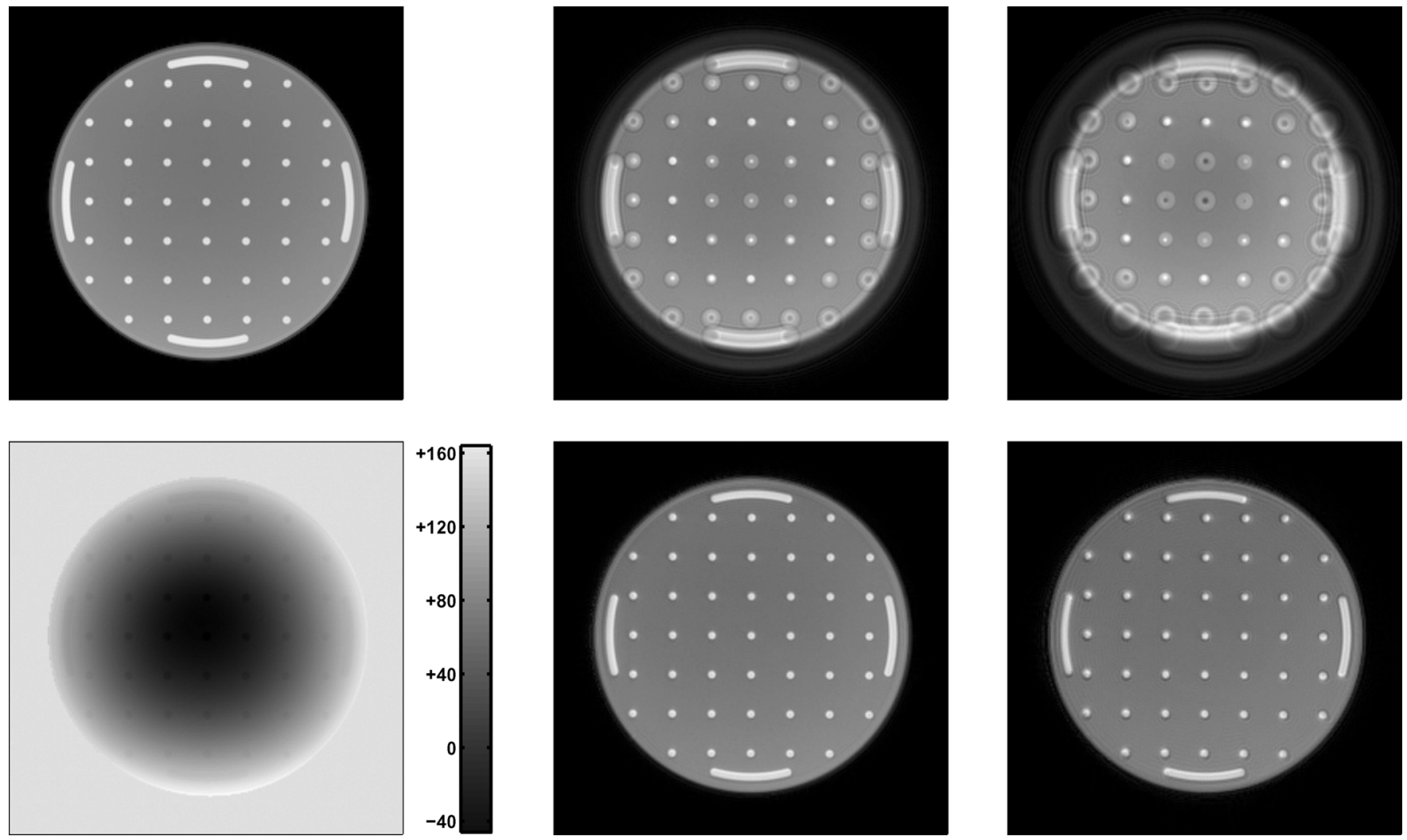

Fig. 4. Results of phantom experiments with the 2-D $\otimes 1$-D NFFT-T approach. Shown are a reference image and a field map obtained with Cartesian $k$-space sampling on the left, and two corresponding uncorrected and corrected images obtained with spiral $k$-space sampling in the middle and on the right. The latter were reconstructed from measurements with a readout duration of $28.5 \mathrm{~ms}$ and $56.5 \mathrm{~ms}$, respectively.

Let a function $\varphi \in L^{2}(\mathbb{R}) \cap L^{1}(\mathbb{R})$, the so-called window function, be given. Its one periodization

$$
\tilde{\varphi}(k):=\sum_{p=-\infty}^{\infty} \varphi(k+p)
$$

is assumed to have a uniformly convergent Fourier series. Hence, it may be written as

$$
\tilde{\varphi}(k)=\sum_{x=-\infty}^{\infty} c_{x}(\tilde{\varphi}) \mathrm{e}^{2 \pi \mathrm{i} k x}
$$

with Fourier coefficients

$$
c_{x}(\tilde{\varphi}):=\int_{-1 / 2}^{1 / 2} \tilde{\varphi}(k) \mathrm{e}^{-2 \pi \mathrm{i} k x} \mathrm{~d} k
$$

where $x \in \mathbb{Z}$. Substituting $k$ by $k-k^{\prime}$ in (A-1) yields

$$
c_{x}(\tilde{\varphi})=\int_{-1 / 2}^{1 / 2} \tilde{\varphi}\left(k-k^{\prime}\right) \mathrm{e}^{-2 \pi \mathrm{i}\left(k-k^{\prime}\right) x} \mathrm{~d} k^{\prime}
$$

which may be approximated by

$$
c_{x}(\tilde{\varphi}) \approx \frac{1}{\alpha N} \sum_{l=-\alpha N / 2}^{\alpha N / 2-1} \tilde{\varphi}\left(k-\frac{l}{\alpha N}\right) \mathrm{e}^{-2 \pi \mathrm{i}\left(k-\frac{l}{\alpha N}\right) x}
$$

for $k \in[-(1 / 2),(1 / 2)]$ and $x=-(N / 2), \ldots,(N / 2)$. The factor $\alpha>1$ is commonly referred to as the oversampling factor. For the sake of simplicity, $N$ and $\alpha N$ are assumed to be even. Provided that all $c_{x}(\tilde{\varphi})$ are nonzero, (A-3) may be rewritten as

$$
\mathrm{e}^{2 \pi \mathrm{i} k x} \approx \frac{1}{\alpha N c_{x}(\tilde{\varphi})} \sum_{l=-\alpha N / 2}^{\alpha N / 2-1} \tilde{\psi}\left(k-\frac{l}{\alpha N}\right) \mathrm{e}^{2 \pi \mathrm{i} \frac{l x}{\alpha N}}
$$

where $\tilde{\varphi}$ has been replaced by $\tilde{\psi}$. The latter is the one periodization of a truncation of $\varphi$ defined by

$$
\psi(k):=\left\{\begin{array}{ll}
\varphi(k) & k \in\left[-\frac{\mu}{\alpha N}, \frac{\mu}{\alpha N}\right] \\
0 & k \notin\left[-\frac{\mu}{\alpha N}, \frac{\mu}{\alpha N}\right] .
\end{array} .\right.
$$

The support of $\psi$ is determined by $2 \mu$, the so-called kernel size. Typically, $\mu \in \mathbb{N}$ is chosen such that $\mu \ll N$. The truncation thus reduces the complexity of the evaluation of the right-hand side of (A-4) considerably. 
The NFFT, i.e., the nonuniform FFT of type 2, evaluates the trigonometric polynomial

$$
f(k):=\sum_{x=-N / 2}^{N / 2-1} \hat{f}_{x} \mathrm{e}^{-2 \pi \mathrm{i} k x}
$$

for $N$ given equispaced samples $\hat{f}_{x}$ at $M$ given nonequispaced positions $k_{j} \in[-(1 / 2),(1 / 2)]$. In matrix-vector notation, it reads

$$
f=A \hat{f}
$$

with

$$
\begin{aligned}
\boldsymbol{f} & :=\left(f_{j}\right)_{j=0, \ldots, M-1} \\
\hat{\boldsymbol{f}} & :=\left(\hat{f}_{x}\right)_{x=-N / 2, \ldots, N / 2-1} \\
\boldsymbol{A} & :=\left(\mathrm{e}^{-2 \pi \mathrm{i} k_{j} x}\right)_{j=0, \ldots, M-1 ; x=-N / 2, \ldots, N / 2-1}
\end{aligned}
$$

where $f_{j}:=f\left(k_{j}\right)$. Applying the approximation (A-4) to (A-5) yields

$$
f_{j} \approx \sum_{l=-\alpha N / 2}^{\alpha N / 2-1} \tilde{\psi}\left(k_{j}-\frac{l}{\alpha N}\right) \sum_{x=-N / 2}^{N / 2-1} \frac{\hat{f}_{x}}{\alpha N c_{-x}(\tilde{\varphi})} \mathrm{e}^{-2 \pi \mathrm{i} \frac{l x}{\alpha N}} .
$$

Accordingly, $\boldsymbol{A}$ may be approximated by $\boldsymbol{B F} \boldsymbol{D}$, where $\boldsymbol{D}$ is a diagonal matrix with entries $\boldsymbol{D}_{x x}=1 / c_{-x}(\tilde{\varphi}), \boldsymbol{F}$ an oversampled Fourier matrix, which includes the factor $1 /(\alpha N)$, and $\boldsymbol{B}$ a sparse matrix with entries $\boldsymbol{B}_{j l}=\tilde{\psi}\left(k_{j}-l /(\alpha N)\right)$.

The adjoint NFFT, i.e., the nonuniform FFT of type 1, evaluates the sum

$$
\sum_{j=0}^{M-1} f_{j} \mathrm{e}^{2 \pi \mathrm{i} k_{j} x}
$$

for $M$ given nonequispaced samples $f_{j}$ at $N$ given equispaced positions $x=-(N / 2), \ldots,(N / 2)-1$. It may be considered as a multiplication of $\boldsymbol{f}$ with $\boldsymbol{A}^{\mathrm{H}} \approx \boldsymbol{D}^{\mathrm{H}} \boldsymbol{F}^{\mathrm{H}} \boldsymbol{B}^{\mathrm{H}}$. As pointed out in [7], [18], gridding reconstruction is simply a fast algorithm for the application of $\boldsymbol{D}^{\mathrm{H}} \boldsymbol{F}^{\mathrm{H}} \boldsymbol{B}^{\mathrm{H}}$ to a vector of nonequispaced samples. Including a sampling density compensation, it performs the following [6]

1) a weighting of the data, i.e., a multiplication with a diagonal matrix $W$,

2) a convolution with a window function and a resampling to an oversampled Cartesian grid, i.e., a multiplication with $B^{\mathrm{H}}$

3) an inverse FFT, i.e., a multiplication with $\boldsymbol{F}^{\mathrm{H}}$,

4) a deapodization, i.e., a multiplication with $D^{\dot{H}}$.

Obviously, the adjoint NFFT and gridding reconstruction rely on the same approximation and involve very similar processing. Moreover, the Kaiser-Bessel window, or its Fourier transform, is a particularly good choice for $\varphi$ in both cases [18], [19]. More sophisticated approaches based on scaling vectors [20], a minimization of the Frobenius norm of certain error matrices [21], or a min-max interpolation [22] did not prove significantly superior.

\section{APPENDIX B}

In this appendix, the approximation underlying the NFFT and its adjoint (A-4) is generalized to real $x$. Starting from

$$
\hat{\varphi}(x):=\int_{-\infty}^{\infty} \varphi(k) \mathrm{e}^{-2 \pi \mathrm{i} k x} \mathrm{~d} k
$$

instead of (A-1) leads to

$$
\hat{\varphi}(x)=\int_{-1 / 2}^{1 / 2} \sum_{p=-\infty}^{\infty} \varphi(k+p) \mathrm{e}^{-2 \pi \mathrm{i}(k+p) x} \mathrm{~d} k
$$

and, with the same steps as from (A-2) to (A-4), to

$\mathrm{e}^{2 \pi \mathrm{i} k x} \approx \frac{1}{\alpha N \hat{\varphi}(x)} \sum_{l=-\alpha N / 2}^{\alpha N / 2-1} \sum_{p=-\infty}^{\infty} \psi\left(k-\frac{l}{\alpha N}+p\right) \mathrm{e}^{2 \pi \mathrm{i}\left(\frac{l}{\alpha N}+p\right) x}$

for $k \in[-(1 / 2),(1 / 2)]$ and $x \in[-(N / 2),(N / 2)]$. Like (A-4), this approximation may be reduced to

$$
\mathrm{e}^{2 \pi \mathrm{i} k x} \approx \frac{1}{\alpha N \hat{\varphi}(x)} \sum_{l=-\alpha N / 2}^{\alpha N / 2-1} \psi\left(k-\frac{l}{\alpha N}\right) \mathrm{e}^{2 \pi \mathrm{i} \frac{l x}{\alpha N}}
$$

for $k \in[-(1 / 2)+(\mu / \alpha N),(1 / 2)-(\mu / \alpha N)]$, since the support of $\psi$ is $[-(\mu / \alpha N),(\mu / \alpha N)]$. Consequently, (B-1) is a good approximation if $k x \in[-(N / 4)+(\mu / 2 \alpha),(N / 4)-(\mu / 2 \alpha)]$. It is worth noting that the further restriction of $k$ may in principle be avoided by explicitly taking the periodization into account. This alternative is not explored in the present work, however.

\section{REFERENCES}

[1] D. C. Noll, C. H. Meyer, J. M. Pauly, D. G. Nishimura, and A. Macovski, "A homogeneity correction method for magnetic resonance imaging with time-varying gradients," IEEE Trans. Med. Imag., vol. 10, no. 4, pp. 629-637, Dec. 1991

[2] Y. M. Kadah and X. Hu, "Algebraic reconstruction for magnetic resonance imaging under $B_{0}$ inhomogeneity," IEEE Trans. Med. Imag., vol. 17 , no. 3, pp. 362-370, Jun. 1998.

[3] D. C. Noll, J. M. Pauly, C. H. Meyer, D. G. Nishimura, and A. Macovski, "Deblurring for non-2-D Fourier transform magnetic resonance imaging," Magn. Reson. Med., vol. 25, pp. 319-333, 1992.

[4] L.-C. Man, J. M. Pauly, and A. Macovski, "Multifrequency interpolation for fast off-resonance correction," Magn. Reson. Med., vol. 37, pp. 785-792, 1997.

[5] B. P. Sutton, D. C. Noll, and J. A. Fessler, "Fast, iterative image reconstruction for MRI in the presence of field inhomogeneities," IEEE Trans. Med. Imag., vol. 22, no. 2, pp. 178-188, Feb. 2003.

[6] J. D. O'Sullivan, "A fast sinc function gridding algorithm for Fourier inversion in computer tomography," IEEE Trans. Med. Imag., vol. 4, pp. 200-207, 1985.

[7] G. E. Sarty, R. Bennett, and R. W. Cox, "Direct reconstruction of nonCartesian k-space data using a nonuniform Fast Fourier Transform," Magn. Reson. Med, vol. 45, pp. 908-915, 2001.

[8] J.-Y. Lee and L. Greengard, "The type 3 nonuniform FFT and its applications," J. Comput. Phys., vol. 206, pp. 1-5, 2005.

[9] A. Dutt and V. Rokhlin, "Fast Fourier Transforms for nonequispaced data," SIAM J. Sci. Comput., vol. 14, pp. 1368-1393, 1993. 
[10] B. Elbel and G. Steidl, "Fast Fourier Transforms for nonequispaced data," in Approximation Theory IX, C. K. Chui and L. L. Schumaker, Eds. Nashville, TN: Vanderbilt Univ. Press, 1998, pp. 39-46.

[11] R. D. Hoge, R. K. Kwan, and G. B. Pike, "Density compensation functions for spiral MRI," Magn. Reson. Med., vol. 38, pp. 117-128, 1997.

[12] H. Schomberg, "Off-resonance correction of MR images," IEEE Trans. Med. Imag., vol. 18, no. 6, pp. 481-495, Jun. 1999.

[13] S. Kunis and D. Potts, NFFT 2.0, C Subroutine Library Apr. 2005. [Online]. Available: http://www.tu-chemnitz.de/-potts/nfft

[14] T. B. Harshbarger and D. B. Twieg, "Iterative reconstruction of singleshot spiral MRI with off-resonance," IEEE Trans. Med. Imag., vol. 18, no. 3, pp. 196-205, Mar. 1999.

[15] J. A. Fessler, S. Lee, V. T. Olafsson, H. R. Shi, and D. C. Noll, "Toeplitz-based iterative image reconstruction for MRI with correction for magnetic field inhomogeneity," IEEE Trans. Signal Process., vol. 53, no. 9, pp. 3393-3402, Sep. 2005.

[16] H. Eggers, P. Boemert, and P. Boesiger, "Comparison of gridding- and convolution-based iterative reconstruction algorithms for sensitivityencoded non-Cartesian acquisitions," in Proc. ISMRM, 2002, p. 743.
[17] D. C. Noll, J. A. Fessler, and B. P. Sutton, "Conjugate phase MR reconstruction with spatially variant sample density correction," IEEE Trans. Med. Imag., vol. 24, no. 3, pp. 325-336, Mar. 2005.

[18] D. Potts, G. Steidl, and M. Tasche, "Fast Fourier Transforms for non-equispaced data: A tutorial," in Modern Sampling Theory: Mathematics and Applications, J. J. Benedetto and P. J. S. G. Ferreira, Eds. Boston, MA: Birkhaeuser, 2001, pp. 247-270.

[19] J. I. Jackson, C. H. Meyer, D. G. Nishimura, and A. Macovski, "Selection of a convolution function for Fourier inversion using gridding," IEEE Trans. Med. Imag., vol. 10, no. 3, pp. 473-478, Sep. 1991.

[20] N. Nguyen and Q. H. Liu, "The regular Fourier matrices and nonuniform Fast Fourier Transforms," SIAM J. Sci. Comput., vol. 21, pp. 283-293, 1999.

[21] A. Nieslony and G. Steidl, "Approximate factorizations of Fourier matrices with nonequispaced knots," Linear Algebra Appl., vol. 266, pp. 337-351, 2003.

[22] J. A. Fessler and B. P. Sutton, "Nonuniform Fast Fourier Transforms using min-max interpolation," IEEE Trans. Signal Process., vol. 51, no. 2, pp. 560-574, Feb. 2003. 